\title{
Harms and Benefits of Using Aspirin for Primary Prevention of Cardiovascular Disease: A Narrative Overview
}

\author{
Giuseppe Lippi, MD ${ }^{1, *}$ Elisa Danese, PhD ${ }^{1, *}$ Emmanuel J. Favaloro, PhD, FFSc (RCPA) ${ }^{2}$ \\ ${ }^{1}$ Section of Clinical Biochemistry, University of Verona, Verona, Italy \\ 2 Department of Haematology, Sydney Centres for Thrombosis and \\ Haemostasis, Institute of Clinical Pathology and Medical Research, \\ NSW Health Pathology, Westmead Hospital, Westmead, New South \\ Address for correspondence Giuseppe Lippi, MD, Section of Clinical \\ Biochemistry, University Hospital of Verona, Piazzale LA Scuro, 37134 \\ Verona, Italy (e-mail: giuseppe.lippi@univr.it).
} Wales, Australia

Semin Thromb Hemost 2019;45:157-163.

\begin{abstract}
Aspirin is one of the most often used drugs for prevention and treatment of a variety of thrombotic disorders. This narrative review aims to provide an overview of evidence highlighting potential benefits and relative harms of aspirin in primary prevention of cardiovascular disease. The authors summarize key findings of the ASPirin in Reducing Events in the Elderly (ASPREE) Investigator Group randomized trial and also provide a comparative overview of recent meta-analyses. Overall, all-cause mortality was largely heterogeneous, with some meta-analyses showing a modestly decreased risk in patients taking aspirin, with others reporting no effects, but the ASPREE Investigator Group trial evidencing 14\% higher risk. Regarding cardiovascular disease, the most favorable impact could be noted for major adverse cardiovascular events, with most meta-analyses reporting a decreased risk in people receiving aspirin. Conversely, the ASPREE Investigator Group trial demonstrated no significant impact of aspirin on risk of cardiovascular mortality or ischemic stroke. A modest favorable effect of aspirin in decreasing the risk of myocardial infarction was noted in two meta-analyses, but not in

Keywords

- aspirin

- cardiovascular disease

- mortality

- cancer

- prevention other reports or in the ASPREE Investigator Group trial. Furthermore, one meta-analysis reported a lower risk of future cancer, others failed to report a significant effect, and the ASPREE Investigator Group trial reported a 31\% increased risk. Unlike these conflicting outcomes, the bleeding risk of patients receiving aspirin was found to be consistently enhanced in all reports reviewed. These recent findings would lead us to conclude that the harms of aspirin in primary prevention of cardiovascular disease may be larger than the benefits, especially in the elderly general population.
\end{abstract}

Aspirin is one of the most commonly used drugs worldwide and the first nonsteroidal anti-inflammatory drug to have been discovered. The history of aspirin can be traced back to more than 3,500 years, when bark from the willow tree was used as a pain reliever and antipyretic by ancient Sumerians

These authors have equally contributed to this work.

published online October 22, 2018
Issue Theme Recent Advances in Thrombosis and Hemostasis-Part IV; Guest Editor: Sam Schulman, MD, PhD. and Egyptians. The active compound in willow bark, salicin, was identified and isolated for the first time in 1828 by Johann Buchner and then refined into yellow needle-like crystals 1 year later by Henri Leroux. In 1838, Raffaele Piria, an Italian chemist working at the Sorbonne in Paris, split salicin into a sugar and an aromatic component (salicylaldehyde) and converted the latter into an acid, thus obtaining salicylic acid. ${ }^{1}$

Copyright $\odot 2019$ by Thieme Medical Publishers, Inc., 333 Seventh Avenue, New York, NY 10001, USA. Tel: +1(212) 584-4662.
DOI https://doi.org/ 10.1055/s-0038-1675380. ISSN 0094-6176. 
In 1852, a French chemist, Charles Gerhardt, modified salicylic acid with the introduction of an acetyl group in place of a hydroxyl group to reduce the gastric irritation linked to chronic use of the drug. Although he was formally the first to create acetylsalicylic acid (ASA), the merit of introducing ASA into the market was awarded to Felix Hoffmann who, in 1899 , convinced Bayer to launch the drug under the brand name of Aspirin: the "A" came from acetyl chloride (the reagent used along with sodium to buffer salicylic acid), the "spir" in Spiraea ulmaria (the plant from which the salicylic acid comes), and the "in" was a familiar ending for medicines. For more than 50 years, ASA was then used for the treatment of fever, pain, and inflammation until 1967, when it was proven to also have antiplatelet effects. ${ }^{2}$

The remarkable discoveries that occurred in subsequent years, including the discovery of cyclooxygenase (COX) isoenzymes, thromboxane A2, and prostaglandins (PGs) as well as the development of light transmission aggregometry, represented essential steps for clarifying the mechanism of action of ASA, which was finally proven in 1976 , over 70 years after its original commercialization. ${ }^{3}$ Briefly, ASA permanently inactivates the COX activity of PG H synthase- 1 and PGH synthase-2, also referred to as COX-1 and COX-2. These isoenzymes catalyze the conversion of arachidonic acid into $\mathrm{PGH} 2$, which is the immediate precursor of PGI2 (prostacyclin) and thromboxane (TXA2). ASA acts by selectively acetylating the hydroxyl group of a serine residue (Ser529 in human COX-1, Ser516 in human COX-2). This leads to a steric block, which ultimately prevents binding of arachidonic acid to its catalytic site. Acetylation leads to irreversible COX inhibition. As a consequence, a new enzyme must be synthesized before other prostanoids can be released. ${ }^{3}$ The major effects of ASA are therefore expressed on nonnuclear cells, such as platelets. Inhibition of COX-1-mediated TXA2 production in platelets can be effectively achieved with a low dose of ASA. Since approximately $10 \%$ of the platelet pool is exchanged daily, once-a-day dosing of ASA can efficiently sustain the almost complete inhibition of platelet TXA2 production. Conversely, COX-2-mediated PGI2 production in vascular endothelial cells necessitates larger doses of aspirin and a much shorter dosing interval, because nucleated cells rapidly resynthesize the enzyme. ${ }^{3}$ This explains the nearly 10 -fold difference in daily dose of ASA when it used as an anti-inflammatory rather than as antiplatelet agent.

The anti-inflammatory effects of aspirin are not only attributable to covalent binding of the acetyl group to serine residues of COX enzymes, but the mechanisms of action of this molecule are far from being completely elucidated, though some recent evidence has been provided. ${ }^{4}$ First, sodium salicylate suppresses COX-2 gene transcription induced by interleukin $1 \beta$ by inhibiting the binding of the transcription factor CCAAT/enhancer-binding protein $\beta$ to its promoter region of COX-2. ${ }^{5}$ Then, salicylic acid is capable of preventing the activation of genes involved in the pathogenesis of the inflammatory response, through blocking activation by nuclear factor kappa-light-chain-enhancer of activated B cells (NF-kB) ${ }^{6,7}$ After oral administration, ASA is rapidly absorbed in the stomach and upper intestine. Peak plasma levels typi- cally occur 30 to 40 minutes after ingestion, and inhibition of platelet activity can be observed with platelet functional tests within 1 hour. The use of enteric-coated ASA slows the bioavailability of the drug, which then takes 3 to 4 hours to reach peak plasma levels.

These important biological features have contributed to make aspirin one of the most used drugs for the prevention and management of many thrombotic disorders. Its role in secondary prevention of arterial occlusive disorders (including treatment of patients with increased cardiovascular risk) is now well established, ${ }^{8}$ and emerging evidence also attests that it may play some role in thromboprophylaxis and treatment of venous thrombosis. ${ }^{9,10}$ Nevertheless, several doubts remain as to whether its use may be entirely beneficial for primary prevention of cardiovascular disease. Therefore, this article aims to provide a narrative overview on the putative cardiovascular or noncardiovascular benefits and the associated risks of using aspirin as primary prevention of cardiovascular disease.

\section{Recent Epidemiological Evidence}

The results of a recent randomized, double-blind, placebocontrolled trial, published by the ASPirin in Reducing Events in the Elderly (ASPREE) Investigator Group, shed some light on the still debated issue of using aspirin in primary prevention of cardiovascular disease. ${ }^{11-13}$ The study results were split within three articles, all published in the New England Journal of Medicine and based on 19,114 community-dwelling elderly adults aged 65 years or older, who were randomized to receive either aspirin (100 mg/day) or placebo, and who were then followed up for a median period of 4.7 years.

In the first article, ${ }^{11}$ the composite incidence of mortality, dementia, and chronic physical impairment was found to be virtually identical between the aspirin and placebo groups ( $2.15 \%$ vs. $2.12 \%$ per 1,000 person-years; hazard ratio [HR], $1.01 ; 95 \%$ confidence interval [CI], 0.92-1.11). Even more interesting evidence could be garnered from the analysis of single endpoints, in that aspirin administration was associated with $14 \%$ higher risk of mortality from any cause (HR, 1.14; 95\% CI, 1.01-1.29) and 38\% higher risk of major bleeding events (HR, 1.38; 95\% CI, 1.18-1.62), while no difference was found between aspirin and placebo in the risk of developing both persistent physical disability ( $\mathrm{HR}, 0.85 ; 95 \% \mathrm{CI}$, $0.70-1.03$ ) and dementia (HR, 0.98; 95\% CI, 0.83-1.15).

In the second article from the ASPREE Investigator Group trial, ${ }^{12}$ no difference could also be noted for the incidence of cardiovascular disease events during follow-up between the aspirin and placebo groups (1.07\% vs. $1.13 \%$ person-years; $\mathrm{HR}, 0.95 ; 95 \% \mathrm{CI}, 0.83-1.08)$. The analysis of the single endpoints also failed to show any potential benefit of aspirin, wherein the rate of major adverse cardiovascular event (MACE; HR, 0.89; 95\% CI, 0.77-1.03), fatal cardiovascular disease (HR, 0.97; 95\% CI, 0.71-1.33), hospitalization for heart failure (HR, 1.07; 95\% CI, 0.79-1.44), fatal or nonfatal myocardial infarction (HR, 0.93; 95\% CI, 0.76-1.15), and fatal or nonfatal stroke (HR, $0.89 ; 95 \%, 0.71-1.11)$ did not appear significantly different between the aspirin and placebo cohorts. Among the various bleeding endpoints, aspirin 
usage was associated with a significantly enhanced risk of developing any form of intracranial bleeding (HR, 1.50; 95\% CI, 1.11-2.02), subdural or extradural hemorrhage (HR, 1.79; 95\% CI, 1.06-3.02), and upper gastrointestinal bleeding (HR, 1.87; 95\% CI, 1.32-2.66), while fatal bleeding was comparable between groups (HR, 1.18; 95\% CI, 0.68-2.03).

In the third study from the ASPREE Investigator Group, ${ }^{13}$ the major causes of death that occurred during the 4.7 years median follow-up period were more intensely investigated in the two groups, namely, those receiving $100 \mathrm{mg} /$ day aspirin or placebo. Interestingly, and somewhat unexpectedly, albeit the number of deaths for cardiovascular disease was found to be similar between groups ( $\mathrm{HR}, 0.82 ; 95 \% \mathrm{CI}, 0.62-1.08$ ), people receiving aspirin had a nearly $30 \%$ higher risk of dying from any type of cancer (HR, 1.31; 95\% CI, 1.10-1.56). A subgroup analysis of cancer deaths revealed that aspirin intake was significantly associated with an enhanced risk of mortality from colorectal cancer (HR, 1.77; 95\% CI, 1.02-3.06), while the death rate for other cancers was substantially similar between people taking aspirin or placebo. The risk of dying from cancer in people on aspirin therapy was also found to be higher in men than in women (HR, 1.19; 1.01-1.40), in those who had never used aspirin before enrolment (HR, 1.19; 95\% CI, 1.05-1.36), in nonfrail individuals ( $\mathrm{HR}, 1.26$; $95 \% \mathrm{CI}, 1.04-1.53)$, and in the obese population (HR, $1.38 ; 95 \% \mathrm{CI}, 1.08-1.77$ ).

\section{Additional Recent Epidemiological Evidence}

Although the important findings published by the ASPREE Investigator Group would lead us to conclude that the use of aspirin for primary prevention of cardiovascular disease in the elderly population would generate more harms than benefits, they need to be assessed against previous epidemiological evidence, such as that reported in some very recent meta-analyses, whose findings will be briefly summarized in the following part of this section.

\section{Relationship between Aspirin in Primary Prevention and Cardiovascular Events}

One of the largest systematic reviews on prophylactic use of aspirin in primary prevention of cardiovascular disease was published by Sutcliffe et al in 2013. ${ }^{14}$ Overall, the authors included 27 randomized controlled trials, systematic reviews, and meta-analyses in their analysis, and concluded that albeit a modest lower risk of MACE could be noted (relative risk [RR], 0.90; 95\% CI, 0.85-0.96), aspirin had no significantly favorable effects in lowering the total risk of coronary heart disease (RR, 0.85; 95\% CI, 0.69-1.06).

Guirguis-Blake et al performed a meta-analysis of 11 randomized controlled trials regarding the efficacy of aspirin in primary prevention of cardiovascular events. ${ }^{15}$ Overall, aspirin intake was found to effectively lower the risk of myocardial infarction (RR, 0.78; 95\% CI, 0.71-0.87), but not that of nonfatal stroke (RR, $0.95 ; 95 \% \mathrm{CI}, 0.85-1.06$ ) or cardiovascular death (RR, 0.94; 95\% CI, 0.86-1.03).

Lotrionte et al performed a meta-analysis including 11 randomized trials and totaling 104,101 subjects randomized to placebo or aspirin administration as primary prevention, who were followed-up for a median period of 60 months. ${ }^{16}$ Interestingly, aspirin usage was associated with $15 \%$ lower risk of developing MACE during follow-up (RR, 0.85; 95\% CI, 0.790.92), a favorable effect that was especially evident in people taking aspirin < $100 \mathrm{mg} /$ day ( $\mathrm{RR}, 0.86 ; 95 \% \mathrm{CI}, 0.76-0.97$ ) or $>100 \mathrm{mg} /$ day (RR, $0.80 ; 95 \% \mathrm{CI}, 0.72-0.89$ ), than in those taking aspirin $100 \mathrm{mg} /$ day (RR, 1.02; 95\% CI, 0.85-1.24).

Raju et al recently concluded in an update of their previous meta-analysis, now including 10 randomized controlled trials and totaling 114,734 participants randomized to receive aspirin or no aspirin for primary prevention of cardiovascular disease, ${ }^{17}$ that aspirin usage was effective for preventing MACE (RR, 0.88; 95\% CI, 0.83-0.94) and myocardial infarction (RR, $0.78 ; 95 \% \mathrm{CI}, 0.65-0.94)$, but did not modify the risk of all-cause stroke (RR, $0.93 ; 95 \% \mathrm{CI}, 0.82-1.05$ ) or cardiovascular mortality (RR, 0.96; 95\% CI, 0.84-1.09).

Unlike these findings, Lei et al performed a meta-analysis of 14 randomized trials exploring the effects of aspirin in primary prevention of ischemic stroke in people with or without cardiovascular risk factors. ${ }^{18}$ Overall, aspirin was found to be effective for lowering the risk of ischemic stroke (odds ratio [OR], 0.83; 95\% CI, 0.74-0.93), and this benefit remained virtually identical when the analysis was limited to apparently healthy individuals (OR, 0.83; 95\% CI, 0.74-0.94).

Interesting evidence also emerged from the meta-analysis of Kunutsor et al, who pooled data obtained in 10 randomized trials in diabetic subjects receiving aspirin, placebo, or no treatment. $^{19}$ Although aspirin use was associated with a borderline significant lower risk of MACE (RR, 0.90; 95\% CI, 0.81-0.99), no favorable effect was noted for other specific endpoints, including myocardial infarction (RR, 0.84; 95\% CI, 0.64-1.11), coronary heart disease (RR, 0.98; 95\% CI, 0.791.21 ), stroke (RR, 0.86; 95\% CI, 0.69-1.08), and all cardiovascular deaths (RR, 0.94; 95\% CI, 0.71-1.26). Overall, these findings are aligned to those earlier published by other groups. Calvin et al performed a meta-analysis of nine randomized controlled trials aimed to compare the putative benefits of aspirin for primary prevention of cardiovascular events in patients with or without diabetes. ${ }^{20}$ Even in this meta-analysis no clear favorable effect could be noted in diabetic subjects regarding the risk of myocardial infarction (RR, 1.19; 95\% CI, 0.82-1.17) and ischemic stroke (RR, 0.70; 95\% CI, 0.25-1.97). More recently, Saito et al performed a randomized trial exploring the effect of low-dose aspirin on future cardiovascular events in type 2 diabetes patients who were followed-up for a median period of 10.3 years. ${ }^{21}$ In agreement with previous evidence, low-dose aspirin was almost ineffective in lowering the risk of all cardiovascular events (HR, 1.14; $95 \% \mathrm{CI}$, 0.91-1.42), nonfatal myocardial infarction (HR, 1.26; $95 \% \mathrm{CI}$, $0.70-2.29$ ), and nonfatal ischemic stroke (RR, 1.02; 95\% CI, $0.66-1.58$ ), but considerably enhanced the risk of developing gastrointestinal bleeding (RR, 2.11; 95\% CI, 1.06-4.18).

\section{Relationship between Aspirin in Primary Prevention and in Risk of Bleeding}

Unlike data reported on cardiovascular outcomes, the evidence that primary prevention of cardiovascular disease with aspirin may be associated with a substantial risk of 
bleeding is more obvious and consistent throughout the recent scientific literature.

In the meta-analysis of Sutcliffe et al, ${ }^{14}$ patients receiving aspirin were found to have a substantially higher risk of developing gastrointestinal bleeding (RR 1.37; 95\% CI 1.151.62), major bleeding (RR 1.62; 95\% CI, 1.31-2.00), and even hemorrhagic stroke (RR 1.38; 95\% CI, 1.01-1.82).

Whitlock et al meta-analyzed the results of 10 cardiovascular disease primary prevention trials, totaling 103,787 subjects randomized to receive aspirin or standard therapy, and who were followed-up for a median period of 6 years. ${ }^{22}$ Overall, the risk of gastrointestinal bleeding was found to be substantially enhanced in patients taking aspirin (OR, 1.59; 95\% CI, 1.32-1.91), while that of intracranial bleeding displayed a higher but nonsignificant trend in patients taking aspirin compared with those who did not receive the drug (OR, 1.27; 95\% CI, 0.98-1.66).

In the updated meta-analysis of Raju et al, ${ }^{17}$ aspirin usage for primary prophylaxis of cardiovascular disease was found to be associated with a significantly higher risk of major ( $R R$, 1.66; 95\% CI, 1.41-1.95), gastrointestinal (RR, 1.37; 95\% CI, 1.15-1.62), and intracranial (RR, 1.36; 95\% CI, 1.01-1.82) bleeding.

In the meta-analysis of Lei et al, ${ }^{18}$ aspirin use was found to considerably enhance the risk of hemorrhagic stroke (OR, 1.32; 95\% CI, 1.04-1.68), and this risk also appeared marginally higher when the analysis was limited to apparently healthy individuals (OR, 1.35; 95\% CI, 1.05-1.75).

\section{Relationship between Aspirin in Primary Prevention and Cumulative Death}

In the meta-analysis of Whitlock et al, ${ }^{22}$ overall all-cause mortality was found to be slightly but significantly lower in patients taking aspirin (OR, 0.94; 95\% CI, 0.88-0.99). Identical results were published in another later article from the same team of authors, ${ }^{15}$ although it was also emphasized that this favorable effect was no longer statistically significant when the analysis was limited to trials based on lowdose aspirin (i.e., < 100 mg/day; RR, 0.95; 95\% CI, 0.89-1.01).

Interesting evidence also emerged from the meta-analysis published by Lotrionte et al. ${ }^{16}$ Although the risk of all-cause death was very marginally (but significantly) lower in patients taking aspirin (RR, 0.94; 95\% CI, 0.88-0.99), no clear doseresponse could be noted when a subgroup analysis was performed on different doses ( $<100 \mathrm{mg} /$ day: RR, 0.95; 95\% CI, $0.87-$ 1.03; 100 mg/day: RR, 0.92; 95\% CI, 0.80-1.05; > 100 mg/day; RR, 0.93; 95\% CI, 0.84-1.02).

In the updated meta-analysis of Raju et al, ${ }^{17}$ no statistically significant favorable effect was noted on all-cause mortality (RR, 0.94; 95\% CI, 0.89-1.00).

In the meta-analysis of Kunutsor et $\mathrm{al}^{19}{ }^{19}$ the use of aspirin was not effective to reduce all-cause mortality (RR, 0.94; 95\% CI, 0.83-1.05).

\section{Relationship between Aspirin in Primary Prevention and Cancer Incidence or Mortality}

In the meta-analysis of Whitlock et al, ${ }^{22}$ both cancer incidence (RR, 0.98; 95\% CI, 0.93-1.04) and mortality (RR, 0.96;
95\% CI, 0.87-1.06) were found to be similar in patients taking aspirin or not.

Chubak et al performed a meta-analysis of 20 randomized controlled trials and 4 individual-patient data meta-analyses on the relationship between regular use of aspirin as primary and secondary prevention of cardiovascular disease, ${ }^{23}$ and reported that both overall cancer incidence $(\mathrm{RR}, 0.98 ; 95 \% \mathrm{CI}$, 0.93-1.04) and mortality (RR, 0.96; 95\% CI, 0.87-1.06) were similar in people taking aspirin versus control groups. A favorable outcome was only seen for colorectal cancer, wherein aspirin usage was associated with a $40 \%$ lower incidence (RR, 0.60; 95\% CI, 0.47-0.86) and 33\% lower mortality (RR, 0.67; 95\% CI, 0.52-0.86), an effect that was especially evident 10 to 19 years after aspirin initiation.

In the meta-analysis published by Lotrionte et $\mathrm{al}^{16}{ }^{16}$ neither cancer incidence (RR, 0.98; 95\% CI, 0.93-1.04) nor mortality (RR, 0.97; 95\% CI, 0.88-1.07) were significantly reduced by aspirin usage. This effect was consistent across different aspirin doses regarding both cancer incidence $(<100 \mathrm{mg} /$ day: $\mathrm{RR}, 1.00 ; 95 \%$ CI, 0.93-1.06; 100 mg/day: RR, 0.89; 95\% CI, 0.77-1.03; > 100 mg/day: RR, 1.02; 95\% CI, 0.75-1.39) and cancer death (<100 mg/day: RR, 0.98; 95\% CI, 0.87-1.12; 100 mg/day: RR, 0.90; 95\% CI, 0.71-1.13; > 100 mg/day: RR, 1.00; 95\% CI, 0.80-1.26).

In a subsequent meta-analysis published by Elwood et al, including results of 5 randomized trials and 42 observational studies on low-dose aspirin administration (i.e. $<100 \mathrm{mg} /$ day) in patients diagnosed with cancer, ${ }^{24}$ aspirin usage was associated with $17 \%$ lower cumulative mortality (HR, 0.83; 95\% CI, 0.76-0.90) from three types of frequent cancers (colorectal, breast, and prostate). However, these effects were especially evident in patients with colorectal (HR, 0.80 ; 95\% CI, 0.70-0.92) and prostate ( $\mathrm{HR}, 0.89 ; 95 \% \mathrm{CI}$, $0.79-0.99)$ cancers, but not in those with breast cancer (HR, 0.87; 95\% CI, 0.69-1.09).

More recently, Qiao et al performed a meta-analysis of 218 observational studies, ${ }^{25}$ and concluded that the use of aspirin was associated with a significant lower risk of any type of cancer (RR, 0.89; 95\% CI, 0.87-0.91). Although the definition of aspirin usage was extremely heterogeneous, the major protective effect against the risk of developing malignancies especially concerned esophageal (RR, 0.75; 95\% CI, 0.620.89 ), gastric (RR, $0.75 ; 95 \% \mathrm{CI}, 0.65-0.86)$, colorectal (RR, 0.79; 95\% CI, 0.74-0.85), pancreatic (RR, 0.80; 95\% CI, 0.680.93 ), ovarian (RR, 0.89; 95\% CI, 0.83-0.95), endometrial (RR, 0.92; 95\% CI, 0.85-0.99), breast (RR, 0.92: 95\% CI, 0.88-0.96), and prostate (RR, $0.94 ; 95 \% \mathrm{CI}, 0.90-0.99)$ cancers.

\section{Conclusion}

Although a significant heterogeneity was found in several meta-analyses (the definition of aspirin usage was extremely heterogeneous across different trials, mostly ranging between $75 \mathrm{mg}$ and $1.2 \mathrm{~g}$ per day), the current published evidence on aspirin use as primary prevention for cardiovascular disease suggests that the harms may be larger than the benefits, especially in the elderly general population ( - Table 1). Overall, the impact on all-cause mortality was found to be largely heterogeneous, with some meta-analyses showing a modestly 


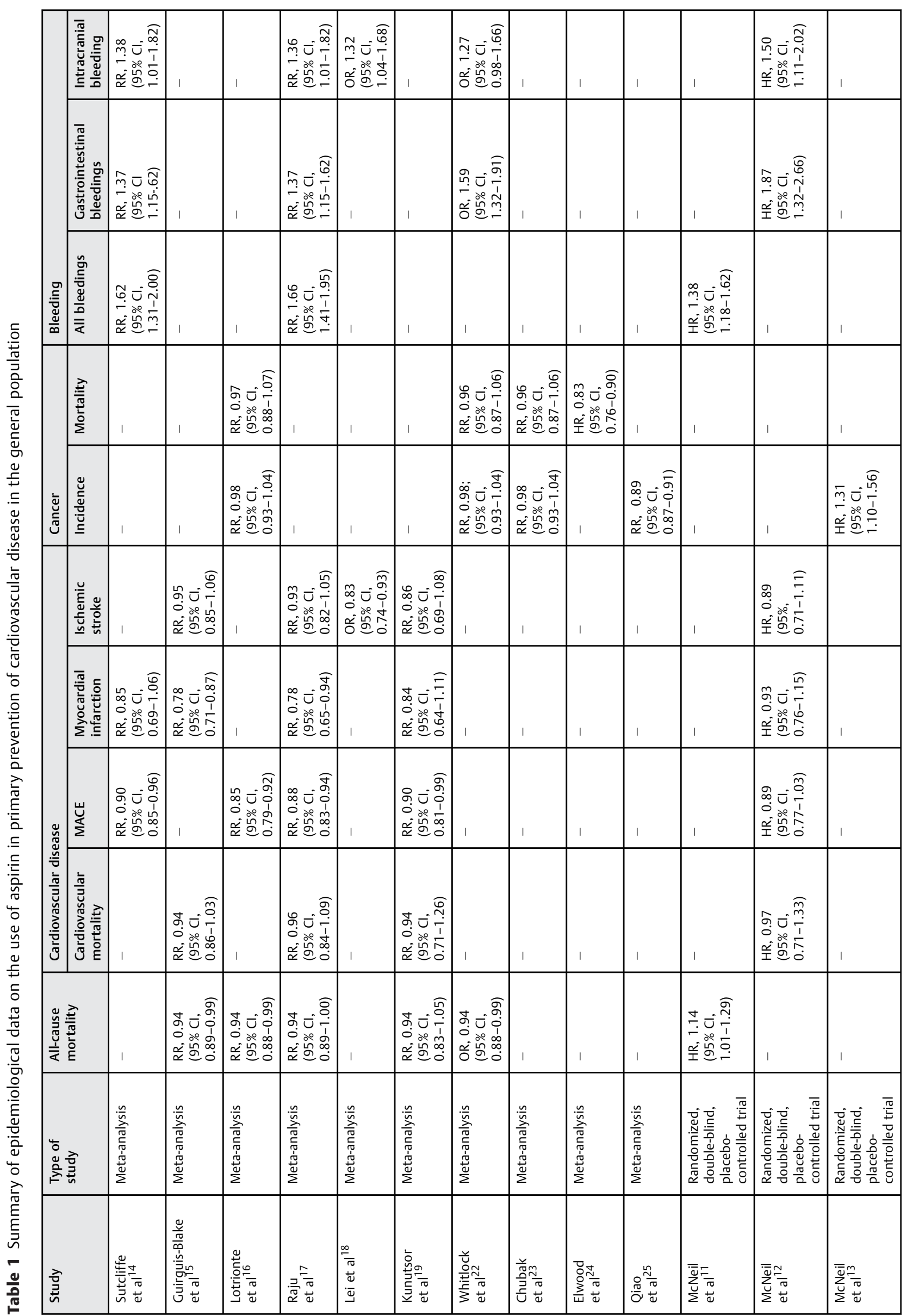

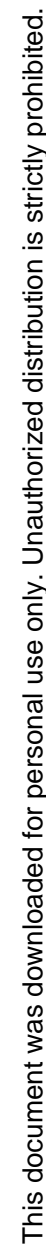


Table 2 Summary of epidemiological data on colon cancer in studies comparing aspirin and placebo in primary prevention of cardiovascular disease in the general population

\begin{tabular}{|l|l|l|l|l|}
\hline Study & Type of study & $\begin{array}{l}\text { Total number } \\
\text { of patients }\end{array}$ & Incidence & Mortality \\
\hline${\text { Chubak et } \mathrm{al}^{23}}$ & Meta-analysis & $\begin{array}{l}8,282 \text { on aspirin and } \\
5,751 \text { on placebo }\end{array}$ & $\begin{array}{l}123 \text { on aspirin and } \\
152 \text { on placebo } \\
(\mathrm{RR}, 0.60 ; 95 \% \mathrm{Cl}, \\
0.47-0.76)\end{array}$ & $\begin{array}{l}119 \text { on aspirin and 121 on placebo } \\
\text { (RR, 0.67; 95\% Cl, 0.52-0.86) }\end{array}$ \\
\hline Elwood et $\mathrm{al}^{24}$ & Meta-analysis & $\mathrm{N} / \mathrm{A}$ & - & $\mathrm{N} / \mathrm{A}(\mathrm{HR}, 0.80 ; 95 \% \mathrm{Cl}, 0.70-0.92)$ \\
\hline Qiao et $\mathrm{al}^{25}$ & Meta-analysis & $\mathrm{N} / \mathrm{A}$ & - & $\mathrm{N} / \mathrm{A}(\mathrm{RR}, 0.79 ; 95 \% \mathrm{Cl}, 0.74-0.85)$ \\
\hline McNeil et al ${ }^{13}$ & $\begin{array}{l}\text { Randomized, } \\
\text { double-blind, } \\
\text { placebo-controlled trial }\end{array}$ & $\begin{array}{l}9,525 \text { on aspirin and } \\
9,589 \text { on placebo }\end{array}$ & - & $\begin{array}{l}35 \text { on aspirin and 20 on placebo } \\
(\mathrm{HR}, 1.31 ; 95 \% \mathrm{Cl}, 1.10-1.56)\end{array}$ \\
\hline
\end{tabular}

Abbreviations: $95 \% \mathrm{Cl}$, 95\% confidence interval; HR, hazard ratio; N/A, not available; RR, relative risk.

decreased risk in patients taking aspirin, ${ }^{15,16,22}$ others reporting no effects, ${ }^{17,19}$ and the very recent randomized trial of the ASPREE Investigator Group even displaying a 14\% higher risk of all-cause death in the aspirin cohort. ${ }^{11}$ Regarding cardiovascular disease, the most favorable impact could be noticed for MACE, with most meta-analyses ${ }^{14,16,17,19}$ (but not the study of the ASPREE Investigator Group), ${ }^{12}$ reporting a decreased risk in people receiving aspirin. However, this apparent benefit does not translate into tangible benefits on reducing the risk of cardiovascular mortality, ${ }^{12,15,17,19}$ or ischemic stroke, ${ }^{12,15,17,19}$ which appears constantly unaffected by the use of aspirin in all published studies, except in the meta-analysis of Lei et al. ${ }^{18} \mathrm{~A}$ modest favorable effect of aspirin in decreasing the risk of myocardial infarction was noted in two meta-analyses, ${ }^{15,17}$ but not in other two meta-analyses, ${ }^{14,19}$ nor in the randomized trial of the ASPREE Investigator Group. ${ }^{12}$

The potential impact of aspirin on future cancer risk also appears largely heterogeneous, wherein one meta-analysis reported a lower risk, ${ }^{25}$ other meta-analyses failed to report a significant effect, ${ }^{16,22,23}$ while the randomized trial of the ASPREE Investigator Group even reported a 31\% increased risk of developing malignancies, especially colorectal cancer (-Table 2).$^{13}$ Similar conclusions can be made for cancerrelated mortality, since only one meta-analysis reported a decreased mortality in patients taking aspirin, ${ }^{24}$ while this finding could not be replicated in the others. ${ }^{16,22,23}$ Unlike these endpoints, the bleeding risk of patients receiving aspirin for primary prevention of cardiovascular disease was found to be consistently increased in all published studies. ${ }^{12,14,17,22}$ In only one meta-analysis was aspirin treatment not associated with intracranial bleeding, ${ }^{22}$ whereas such risk was found to be significantly increased in the remaining reports, ${ }^{14,17,18}$ as well as in the randomized trial of the ASPREE Investigator Group. ${ }^{12}$ Regarding aspirin dosages, no major benefits (or harms) could be observed in studies analyzing the effect of low-dose aspirin (-Table 3).

Taken together, the data reported in recent meta-analyses, combined with the findings of the ASPREE Investigator Group, would lead us to conclude that aspirin, as used for primary prevention of cardiovascular disease, does not provide clear benefits, either in terms of reducing all-cause and cardiovascular mortality, or for lowering the risk of future cardiovascular

Table 3 Summary of epidemiological data in studies comparing different aspirin doses for primary prevention of cardiovascular disease in the general population

\begin{tabular}{|c|c|c|c|c|c|}
\hline \multirow[t]{2}{*}{ Endpoint } & \multicolumn{3}{|l|}{${\text { Lotrionte et } \text { al }^{16}}^{16}$} & \multicolumn{2}{|l|}{ Guirguis-Blake et al ${ }^{15}$} \\
\hline & $<100 \mathrm{mg} / \mathrm{d}$ & $100 \mathrm{mg} / \mathrm{d}$ & $>100 \mathrm{mg} / \mathrm{d}$ & All doses & $<100 \mathrm{mg} / \mathrm{d}$ \\
\hline Mortality & $\begin{array}{l}\text { RR, } 0.95 \\
(95 \% \mathrm{Cl}, 0.87-1.03)\end{array}$ & $\begin{array}{l}\text { RR, } 0.92 \\
(95 \% \mathrm{Cl}, 0.80-1.05)\end{array}$ & $\begin{array}{l}\text { RR, } 0.93 \\
(95 \% \mathrm{Cl}, 0.84-1.02)\end{array}$ & $\begin{array}{l}\text { RR, } 0.94 \\
(95 \% \mathrm{Cl}, 0.89-0.99)\end{array}$ & $\begin{array}{l}\text { RR, } 0.95 \\
(95 \% \mathrm{Cl}, 0.89-1.01)\end{array}$ \\
\hline MACE & $\begin{array}{l}\text { RR, } 0.86 \\
(95 \% \mathrm{Cl}, 0.76-0.97)\end{array}$ & $\begin{array}{l}\text { RR, } 1.02 \\
(95 \% \mathrm{Cl}, 0.85-1.24)\end{array}$ & $\begin{array}{l}\text { RR, } 0.80 \\
(95 \% \mathrm{Cl}, 0.72-0.89)\end{array}$ & $\begin{array}{l}\text { RR, } 0.94 \\
(95 \% \mathrm{Cl}, 0.86-1.03)\end{array}$ & $\begin{array}{l}\text { RR, } 0.97 \\
(95 \% \mathrm{Cl}, 0.85-1.10)\end{array}$ \\
\hline $\begin{array}{l}\text { Cancer } \\
\text { incidence }\end{array}$ & $\begin{array}{l}\text { RR, } 1.00 \\
(95 \% \mathrm{Cl}, 0.93-1.06)\end{array}$ & $\begin{array}{l}\text { RR, } 0.89 \\
(95 \% \mathrm{Cl}, 0.77-1.03)\end{array}$ & $\begin{array}{l}\text { RR, } 1.02 \\
(95 \% \mathrm{Cl}, 0.75-1.39)\end{array}$ & - & - \\
\hline Cancer death & $\begin{array}{l}\mathrm{RR}, 0.98 \\
(95 \% \mathrm{Cl}, 0.87-1.12)\end{array}$ & $\begin{array}{l}\text { RR, } 0.90 \\
(95 \% \mathrm{Cl}, 0.71-1.13)\end{array}$ & $\begin{array}{l}\mathrm{RR}, 1.00 \\
(95 \% \mathrm{Cl}, 0.80-1.26)\end{array}$ & - & - \\
\hline $\begin{array}{l}\text { Intracranial } \\
\text { bleeding }\end{array}$ & $\begin{array}{l}\text { RR, } 1.17 \\
(95 \% \mathrm{Cl}, 0.84-1.63)\end{array}$ & $\begin{array}{l}\text { RR, } 1.32 \\
(95 \% \mathrm{Cl}, 0.63-2.73)\end{array}$ & $\begin{array}{l}\text { RR, } 1.57 \\
(95 \% \mathrm{Cl}, 0.89-2.77)\end{array}$ & - & - \\
\hline $\begin{array}{l}\text { Gastrointestinal } \\
\text { bleeding }\end{array}$ & $\begin{array}{l}\text { RR, } 1.60 \\
(95 \% \text { Cl, 1.29-1.99) }\end{array}$ & $\begin{array}{l}\text { RR, } 1.13 \\
(95 \% \mathrm{Cl}, 0.44-2.91)\end{array}$ & $\begin{array}{l}\text { RR, } 1.59 \\
(95 \% \mathrm{Cl}, 1.02-2.48)\end{array}$ & - & - \\
\hline
\end{tabular}

Abbreviations: $95 \% \mathrm{Cl}$, 95\% confidence interval; MACE, major adverse cardiovascular events; RR, relative risk. 
events. The impact on the risk of developing cancer also remains questionable, even for colorectal cancers, as recently shown by the evidence provided by the ASPREE Investigator Group. On the other hand, these uncertain benefits seem counterbalanced, probably completely overwhelmed, by an increased risk of developing major bleeding, especially from the gastrointestinal tract.

\section{Conflict of Interest}

None.

\section{Disclaimer}

The opinions expressed here are those of the authors, and are not necessarily those of the University Hospital of Verona or of New South Wales Health Pathology.

\section{References}

1 Desborough MJR, Keeling DM. The aspirin story - from willow to wonder drug. Br J Haematol 2017;177(05):674-683

2 Miner J, Hoffhines A. The discovery of aspirin's antithrombotic effects. Tex Heart Inst J 2007;34(02):179-186

3 Vane JR, Botting RM. The mechanism of action of aspirin. Thromb Res 2003;110(5-6):255-258

4 Cadavid AP. Aspirin: the mechanism of action revisited in the context of pregnancy complications. Front Immunol 2017;8:261

5 Saunders MA, Sansores-Garcia L, Gilroy DW, Wu KK. Selective suppression of CCAAT/enhancer-binding protein beta binding and cyclooxygenase-2 promoter activity by sodium salicylate in quiescent human fibroblasts. J Biol Chem 2001;276(22):18897-18904

6 Yin MJ, Yamamoto Y, Gaynor RB. The anti-inflammatory agents aspirin and salicylate inhibit the activity of I(kappa)B kinase-beta. Nature 1998;396(6706):77-80

7 Shi X, Ding M, Dong Z, et al. Antioxidant properties of aspirin: characterization of the ability of aspirin to inhibit silica-induced lipid peroxidation, DNA damage, NF-kappaB activation, and TNFalpha production. Mol Cell Biochem 1999;199(1-2):93-102

8 Baigent C, Blackwell L, Collins R, et al; Antithrombotic Trialists' (ATT) Collaboration. Aspirin in the primary and secondary prevention of vascular disease: collaborative meta-analysis of individual participant data from randomised trials. Lancet 2009;373 (9678):1849-1860

9 Lippi G, Favaloro EJ. Venous and arterial thromboses: two sides of the same coin? Semin Thromb Hemost 2018;44(03):239-248

10 Anderson DR, Dunbar M, Murnaghan J, et al. Aspirin or rivaroxaban for VTE prophylaxis after hip or knee arthroplasty. N Engl J Med 2018;378(08):699-707

11 McNeil JJ, Woods RL, Nelson MR, et al; ASPREE Investigator Group. Effect of aspirin on disability-free survival in the healthy elderly. NEngl J Med 2018; [Epub ahead of print]. Doi: 10.1056/NEJMoa1800722

12 McNeil JJ, Wolfe R, Woods RL, et al; ASPREE Investigator Group. Effect of aspirin on cardiovascular events and bleeding in the healthy elderly. N Engl J Med 2018; [Epub ahead of print]. Doi: 10.1056/NEJMoa 1805819

13 McNeil JJ, Nelson MR, Woods RL, et al; ASPREE Investigator Group. Effect of aspirin on all-cause mortality in the healthy elderly. N Engl J Med 2018; [Epub ahead of print]. Doi: 10.1056/NEJMoa1803955

14 Sutcliffe P, Connock M, Gurung T, et al. Aspirin for prophylactic use in the primary prevention of cardiovascular disease and cancer: a systematic review and overview of reviews. Health Technol Assess 2013;17(43):1-253

15 Guirguis-Blake JM, Evans CV, Senger CA, O'Connor EA, Whitlock EP. Aspirin for the primary prevention of cardiovascular events: a systematic evidence review for the U.S. Preventive Services Task Force. Ann Intern Med 2016;164(12):804-813

16 Lotrionte M, Biasucci LM, Peruzzi M, Frati G, Giordano A, BiondiZoccai G. Which aspirin dose and preparation is best for the longterm prevention of cardiovascular disease and cancer? Evidence from a systematic review and network meta-analysis. Prog Cardiovasc Dis 2016;58(05):495-504

17 Raju N, Sobieraj-Teague M, Bosch J, Eikelboom JW. Updated metaanalysis of aspirin in primary prevention of cardiovascular disease. Am J Med 2016;129(05):e35-e36

18 Lei H, Gao Q Liu SR, Xu J. The benefit and safety of aspirin for primary prevention of ischemic stroke: a meta-analysis of randomized trials. Front Pharmacol 2016;7:440

19 Kunutsor SK, Seidu S, Khunti K. Aspirin for primary prevention of cardiovascular and all-cause mortality events in diabetes: updated meta-analysis of randomized controlled trials. Diabet Med 2017;34 (03):316-327

20 Calvin AD, Aggarwal NR, Murad MH, et al. Aspirin for the primary prevention of cardiovascular events: a systematic review and meta-analysis comparing patients with and without diabetes. Diabetes Care 2009;32(12):2300-2306

21 Saito Y, Okada S, Ogawa H, et al; JPAD Trial Investigators. Lowdose aspirin for primary prevention of cardiovascular events in patients with type 2 diabetes mellitus: 10-year follow-up of a randomized controlled trial. Circulation 2017;135(07): 659-670

22 Whitlock EP, Williams SB, Burda BU, et al. Aspirin Use in Adults: Cancer, All-Cause Mortality, and Harms: A Systematic Evidence Review for the U.S. Preventive Services Task Force [Internet]. Rockville, MD: Agency for Healthcare Research and Quality (US); 2015

23 Chubak J, Whitlock EP, Williams SB, et al. Aspirin for the prevention of cancer incidence and mortality: systematic evidence reviews for the U.S. Preventive Services Task Force. Ann Intern Med 2016;164(12):814-825

24 Elwood PC, Morgan G, Pickering JE, et al. Aspirin in the treatment of cancer: reductions in metastatic spread and in mortality: a systematic review and meta-analyses of published studies. PLoS One 2016;11(04):e0152402

25 Qiao Y, Yang T, Gan Y, et al. Associations between aspirin use and the risk of cancers: a meta-analysis of observational studies. BMC Cancer 2018;18(01):288 